\title{
A New Method for Solving Multiobjective Bilevel Programs
}

\author{
Ying Ji, ${ }^{1}$ Shaojian Qu, ${ }^{1}$ and Zhensheng $Y u^{2}$ \\ ${ }^{1}$ Business School, University of Shanghai for Science and Technology, 516 Jungong Road, Shanghai 200093, China \\ ${ }^{2}$ College of Science, University of Shanghai for Science and Technology, Shanghai, China \\ Correspondence should be addressed to Ying Ji; jiying_1981@126.com
}

Received 14 May 2016; Revised 15 August 2016; Accepted 28 December 2016; Published 23 March 2017

Academic Editor: Kamel Barkaoui

Copyright (C) 2017 Ying Ji et al. This is an open access article distributed under the Creative Commons Attribution License, which permits unrestricted use, distribution, and reproduction in any medium, provided the original work is properly cited.

\begin{abstract}
We study a class of multiobjective bilevel programs with the weights of objectives being uncertain and assumed to belong to convex and compact set. To the best of our knowledge, there is no study about this class of problems. We use a worst-case weighted approach to solve this class of problems. Our "worst-case weighted multiobjective bilevel programs" model supposes that each player (leader or follower) has a set of weights to their objectives and wishes to minimize their maximum weighted sum objective where the maximization is with respect to the set of weights. This new model gives rise to a new Pareto optimum concept, which we call "robust-weighted Pareto optimum"; for the worst-case weighted multiobjective optimization with the weight set of each player given as a polytope, we show that a robust-weighted Pareto optimum can be obtained by solving mathematical programing with equilibrium constraints (MPEC). For an application, we illustrate the usefulness of the worst-case weighted multiobjective optimization to a supply chain risk management under demand uncertainty. By the comparison with the existing weighted approach, we show that our method is more robust and can be more efficiently applied to real-world problems.
\end{abstract}

\section{Introduction}

Multiobjective bilevel programs are a generalization of the scalar criterion bilevel programs [1] and used to model situations where decision-makers at both upper and lower levels, called players, take actions by considering their individual multiple objectives [2-4]. Many papers have been published in the last two decades about (multiobjective) bilevel programs because of their possible applications $[1,4,5]$.

In this paper, we consider a special class of multiobjective bilevel programs with the weights of objectives being uncertain and assumed to belong to convex and compact set. To the best our knowledge, there is no study about this class of problems and the existing approaches in multiobjective bilevel programs can not solve this class of problems. Therefore, new methods are necessary to be proposed. In this paper, we present a robust-weighted approach to solve this class of multiobjective bilevel programs. In our model, we suppose that players at both upper and lower levels are risk-averse and a player uses the robust optimization approach to manage the weight uncertainty, assuming that the other player is a robust optimizer as well. Note that the robust optimization approach is not concerning problem data or the other players' strategies but concerning the weights to the objectives. We note that this model is closely related to robust optimization [6, 7]. It can be seen as a natural extension of the robust modeling technique by replacing a decision variable with a general deterministic function $[6,7]$. However, our model is also different from the studies in $[6,7]$ that we study the bilevel problem, while the model in $[6,7]$ is single level.

Multiobjective optimization has been extensively studied and a number of different approaches have also been proposed [8]. In multiobjective problems, since there are several competing objectives to be considered and it is not possible to simultaneously optimize all objectives, a commonly accepted approach for coping with this setting is the weighted approach by assigning a nonnegative weight by considering the importance of the corresponding objective function. And then the decision-maker can make a decision by optimizing a weighted sum objective [9-12]. A weighted Pareto optimal point can be obtained. In the setting of multiobjective models including input from multiple experts, a decision can often be obtained only after considering different opinions from different experts [11]. 
However current approaches to model-based multiexpert multiobjective decision-making have several shortcomings. How to choose a solution among (possibly infinite) generated Pareto optimal solutions? There is no guidance. In multiexpert optimization, the relative weights given by experts can differ significantly as experts with differing opinions often assign different relative weights to objectives. As shown in applications, the weights are not known in advance and the player has to choose them. Ambiguity often exists in the choice of the weights to objectives, as it is not easy to decide relative weights for each objective. In addition, as shown in multiobjective optimization in the literature, relative weights given by the same decision-maker may rely on the elicitation methods $[12,13]$. In the bilevel setting considered in this paper, it is more difficult for the decision-maker at the upper level to exactly assume the weights of the decision-maker's objectives at the lower level. Therefore, in this paper, we focus on a special class of multiobjective bilevel programs with the weights of objectives being uncertain and assumed to belong to convex and compact set. There is no study about this class of problems and there no methods in multiobjective bilevel programs can solve this class of problems. It is necessary to provide a new approach to cope with these issues.

Hence, our motivation to utilize a worst-case weighted approach is that it provides an alternative way to deal with the weights ambiguity. Furthermore, if each player in multiobjective bilevel problems chooses the worst-case weighted approach, then we show that the computation for robustweighted Pareto optimum, with the choice of polytope weight set for every player, is reformulated as a solution to mathematical programing with equilibrium constraints (MPEC) which can be solved by the existing methods (e.g., the sequential quadratic programing (SQP) methods).

Though multiobjective bilevel programs have not attracted much attention in the literature, there are some interesting potential applications. One example is the multicriteria Stackelberg competition of a supply chain containing a manufacturer who supplies a set of products to a riskaverse retailer satisfying uncertain consumer demand. In our model, the manufacturer decides on the quantity for each product so as to maximize the profit and minimize the cost simultaneously by forecasting the order quantity from the retailer and the wholesale prices resulting from market clearing conditions. The retailer also decides their wholesale market order quantity for each product in order to simultaneously maximize the mean profit and minimize the standard deviation of the profit.

Since the possible applications, multiobjective bilevel programs have attracted some attention. For example, Yin [14] considers a multiobjective bilevel model for transportation planning and management problems, where genetic algorithms are proposed to solve the resulting model. Deb and Sinha [15] present evolutionary algorithms for solving multiobjective optimization problems. Pieume et al. [16] develop two methods for solving bilevel linear multiobjective optimization problems. Eichfelder [17] gives a solution method for solving nonlinear multiobjective bilevel problems based on a scalarization approach and the sensitivity analysis of adaptive parameters. To reduce traffic congestion as well as to improve workforce productivity, a bilevel multiobjective model is proposed for an urban logistics metropolis [18]. A discrete approach is proposed to solve the resulting model. The convergence result and numerical tests are also provided. Some special multiobjective bilevel programs with linear objectives are also studied extensively. For example, a linear bilevel optimization problem with multiple objectives at the upper level is studied in [19], where the original problem reduces to solving a series of linear bilevel problems with a single objective function at each level; a linear bilevel optimization problem with multiple objectives at the lower level is studied in [20], where the original problem is reformulated as an optimization problem over a nonconvex region given by a union of faces of the polyhedron defined by all constraints.

Our study is different from the above papers in that we focus on a special class of multiobjective bilevel nonlinear programs - the weights of objectives are uncertain and assumed to belong to convex and compact set. To deal with this uncertainty, a robust optimization approach is used. Then we reformulated the original problem as a robust bilevel nonlinear optimization problem. To the best of our knowledge, this is the first paper to consider these special multiobjective bilevel problems.

We feel that the primary contributions of this paper are as follows. We deal with a special class of multiobjective bilevel programs, where the weights of objectives are uncertain and assumed to belong to convex and compact set. To the best of our knowledge, this is the first paper to consider such multiobjective bilevel programs. In our model, we suppose that players at both upper and lower levels are risk-averse and a player uses the robust optimization approach to manage the weight uncertainty, assuming that the other player is a robust optimizer as well. As we know that, there is no method to solve such problem. We propose a worst-case weighted approach for solving such multiobjective bilevel programs, extending the notion of robust-weighted multiobjective optimization models [21] to multiobjective bilevel problems. We show that a robust-weighted Pareto optimal point can be calculated by solving MPEC when the weight sets chosen by the players are polytopes. We note that the MPEC has been extensively studied and can be solved by the sequential quadratic programing (SQP) method [22]. We demonstrate the usefulness of the worst-case weighted method in a bilevel multiobjective competition problem within supply chain. We note that, compared with the existing weighted approach [9], our method is more "robust" in that there are different solutions for choosing different weights by using weighted approach [9], but there is a unique solution by using our approach.

Throughout this paper we use the following notations. $R$ is the set of real numbers; $R_{+}$denotes the set of nonnegative real numbers; and $R_{++}$is the set of strictly positive real numbers. Let

$$
\begin{aligned}
R^{m} & =\underbrace{R \times \cdots \times R}_{m} ; \\
R_{+}^{m} & =\underbrace{R_{+} \times \cdots \times R_{+} ;}_{m} \\
R_{++}^{m} & =\underbrace{R_{++} \times \cdots \times R_{++}}_{m} .
\end{aligned}
$$




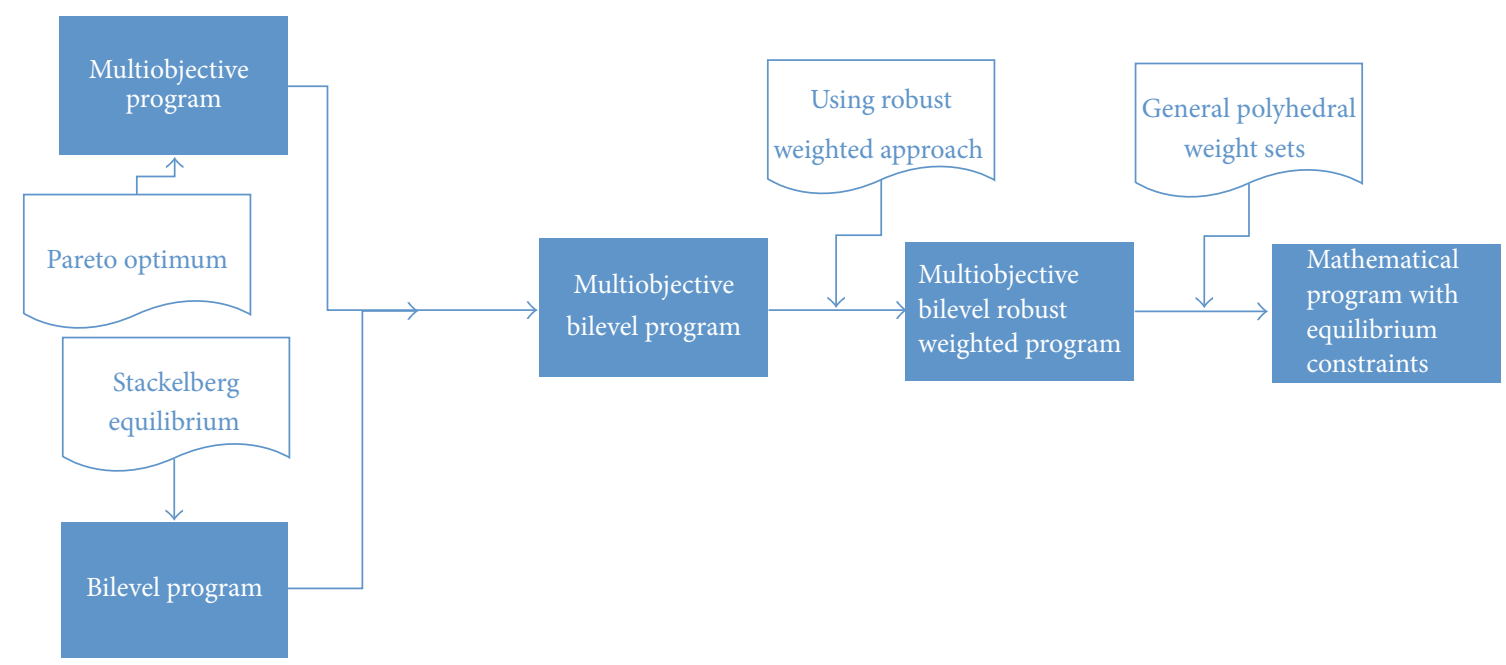

FIGURE 1: Illustration of links about the different mechanisms used.

For any $u, v \in R^{m}$, denote

$$
\begin{aligned}
v & \geq u(\operatorname{resp} . v \leq u) \Longleftrightarrow \\
v-u & \in R_{+}^{m}\left(\operatorname{resp} . u-v \in R_{+}^{m}\right) \Longleftrightarrow \\
v_{j}-u_{j} & \geq 0\left(\operatorname{resp} . v_{j}-u_{j} \leq 0\right), \quad j \in I ; \\
u & >v(\operatorname{resp} . v \prec u) \Longleftrightarrow \\
v-u & \in R_{++}^{m}\left(\operatorname{resp} . u-v \in R_{++}^{m}\right) \Longleftrightarrow \\
v_{j}-u_{j} & >0\left(\operatorname{resp} . v_{j}-u_{j}<0\right), \quad j \in I ; \\
v & \geq u(\operatorname{resp} . v \leq u) \Longleftrightarrow \\
v-u & \in R_{+}^{m}\left(\operatorname{resp} . u-v \in R_{+}^{m}\right), \quad u \neq v \Longleftrightarrow \\
v_{j}-u_{j} & \geq 0\left(\operatorname{resp} . v_{j}-u_{j} \leq 0\right), \quad j \in I, u \neq v,
\end{aligned}
$$

where $I:=\{1, \ldots, m\}$ is the index set. Given any vector function $h: R^{n} \rightarrow R^{m}$, by $h \in C^{1}\left(R^{n}, R^{m}\right)$, we indicate that $h$ is a continuously differentiable function from $R^{n}$ to $R^{m}$ and we use $\nabla h(x) \in R^{n \times m}$ to denote the gradient of the function $h$ at $x$; for simplicity, in this paper we use superscript $U$ (resp., $L$ ) to denote decision-makers in the upper level (resp., decision-makers in the lower level) parameters, decision variables, cost functions, and weights of payoff functions. We note that the different mechanisms (e.g., bilevel, equilibrium, multiobjective optimization, weighting, and Pareto) are used in this paper. To specially understand the links between all these components, we provide a figure (see Figure 1) to illustrate this.

In Section 2, we review some basic concepts in multiobjective bilevel programs and then introduce a new concept of the robust-weighted Pareto optimum. We show that a robustweighted Pareto optimal solution is also a weighted Pareto optimum. In Section 3, we show that when the weight sets are given as polytopes, the robust-weighted Pareto optimum can be cast as MPEC which can be efficiently solved by SQP methods. In Section 4, we show the efficiency of our approach by an example for analyzing a multiobjective bilevel competition problem within supply chain. Finally, we conclude this paper in Section 5.

\section{Preliminaries}

In this paper, we consider the following multiobjective bilevel optimization:

$$
\begin{array}{cl}
\min _{x^{i} \in R^{n^{i}}, i=U, L} & F^{U}\left(x^{U}, x^{L}\right) \\
\text { s.t. } & G^{U}\left(x^{U}, x^{L}\right) \geq 0, \\
& H^{U}\left(x^{U}, x^{L}\right)=0, \\
& x^{L} \in S^{L}\left(x^{U}\right),
\end{array}
$$

where $S^{L}\left(x^{U}\right)$ signifies the set of solutions of the lower-level problem

$$
\begin{array}{ll}
\min _{x^{L} \in R^{n^{L}}} & F^{L}\left(x^{L}, x^{U}\right) \\
\text { s.t. } & G^{L}\left(x^{L}, x^{U}\right) \geq 0, \\
& H^{L}\left(x^{L}, x^{U}\right)=0
\end{array}
$$

and $F^{i}: R^{n^{i}} \times R^{n^{j}} \rightarrow R^{m^{i}}, G^{i}: R^{n^{i}} \times R^{n^{j}} \rightarrow R^{p^{i}}$, and $H^{i}: R^{n^{i}} \times$ $R^{n^{j}} \rightarrow R^{q^{i}}, i, j \in\{U, L\}, i \neq j$. In what follows, we assume that all functions considered in the bilevel problem (3) are continuously differentiable and $m^{i} \geq 2, i=U, L$. Since we consider the case of a multiobjective optimization $\left(m^{L} \geq 2\right)$ at the lower level, given $x^{U}$, the solution set $S^{L}\left(x^{U}\right)$ denotes the Pareto optimal set or weak Pareto optimal set; that is, given any $x^{U}$ and $\bar{x}^{L} \in S^{L}\left(x^{U}\right)$, there is no feasible point $x^{L}$ 
to (4) such that $F^{L}\left(x^{L}, x^{U}\right) \preceq F^{L}\left(\bar{x}^{L}, x^{U}\right)$ or $F^{L}\left(x^{L}, x^{U}\right) \prec$ $F^{L}\left(\bar{x}^{L}, x^{U}\right)$.

Definition 1. $\left(x^{U}, x^{L}\right)$ is said to be feasible for problem (3) if $G^{U}\left(x^{U}, x^{L}\right) \geq 0, H^{U}\left(x^{U}, x^{L}\right)=0$, and $x^{L} \in S^{L}\left(x^{U}\right) .\left(\bar{x}^{U}, \bar{x}^{L}\right)$ is said to be locally Pareto optimal (resp., weak Pareto) for problem (3), if it is feasible for (3) and there exists no other feasible point $\left(x^{U}, x^{L}\right)$ in the neighborhood of $\left(\bar{x}^{U}, \bar{x}^{L}\right)$ such that $F^{U}\left(x^{U}, x^{L}\right) \preceq F^{U}\left(\bar{x}^{U}, \bar{x}^{L}\right)$ (resp., $F^{U}\left(x^{U}, x^{L}\right) \prec$ $\left.F^{U}\left(\bar{x}^{U}, \bar{x}^{L}\right)\right)$.

In the multiobjective optimization literature, the weighted approach is one of the most often used methods. However the weighted approach is also criticized for several shortcomings. For example, as shown in applications, weights are not known in advance and the modeler or decision-maker has to choose them while it is often difficult to make a reasonable decision for choosing appropriate weights for objectives. In many situations there may be uncertainty in the weights provided by the decision-maker. Therefore there is a need for providing a novel methodology to cope with these issues. The robust-weighted approach is one possible choice as it provides an alternative to solve the weight uncertainty in the multiobjective bilevel model. Specifically, we assume that players in both levels use the robust-weighted approach to address the weight uncertainty; that is, both the leader and the follower use a robust-weighted approach to minimize their weighted payoff functions.

Definition 2. Let $W^{i} \in W_{r}^{i}:=\left\{w^{i} \in R_{+}^{m^{i}} \mid\left\|w^{i}\right\|_{1}=1\right\}$ be a nonempty closed convex set of weights, $i=U, L$. $\left(x^{U}, x^{L}\right)$ is called a robust-weighted optimal solution of (3), if it is an optimum to the following robust optimization problem:

$$
\begin{aligned}
\min _{x^{i} \in R^{n^{i}}, i=U, L} \max _{w^{U} \in W^{U}} & \left(w^{U}\right)^{T} F^{U}\left(x^{U}, x^{L}\right) \\
\text { s.t. } & G^{U}\left(x^{U}, x^{L}\right) \geq 0, \\
& H^{U}\left(x^{U}, x^{L}\right)=0, \\
& x^{L} \in S_{w}^{L}\left(x^{U}\right),
\end{aligned}
$$

where $S_{w}^{L}\left(x^{U}\right)$ signifies the set of solutions of the lower-level problem:

$$
\begin{aligned}
\min _{x^{L} \in R^{n^{L}} \max _{w^{L} \in W^{L}}} & \left(w^{L}\right)^{T} F^{L}\left(x^{L}, x^{U}\right) \\
\text { s.t. } & G^{L}\left(x^{L}, x^{U}\right) \geq 0, \\
& H^{L}\left(x^{L}, x^{U}\right)=0 .
\end{aligned}
$$

Different from weighted Pareto concepts, the robustweighted optimum proposed in this paper seeks to find an optimal solution that is robust and feasible for the worst-case weight within the family of weights. It directly follows from Theorem 2.2 of $\mathrm{Hu}$ and Mehrotra [21] and Definitions 1 and 2 that the following theorem holds.
Theorem 3. Let $W^{i} \subset W_{r}^{i}$ be defined as in Definition 2, $i=$ $U, L$. if $\bar{x}:=\left(\bar{x}^{U}, \bar{x}^{L}\right)$ is a robust-weighted optimum of (5) with the combination $W:=\left(W^{U}, W^{L}\right)$ of weight sets, then we have the following results:

(1) $\bar{x}$ is a weak Pareto optimum of (3).

(2) if all of the weights in $W^{U}$ are positive, then $\bar{x}$ is a Pareto optimum of (3).

(3) if $\bar{x}$ is the unique optimal solution of (5), then $\bar{x}$ is a Pareto optimum of (3).

\section{MPEC Reformulations for (5)}

Now that a robust-weighted optimum of (5) is a (weak) Pareto optimum of (3), our next step is to discuss how to realize such a solution. To do this, we assume that the weight sets $W^{i}$, $i=U, L$, are given as polyhedral regions. Then the problem of computing a robust-weighted optimal point could be cast as MPEC which has been extensively studied and can be solved by the solver PATH [23].

We suppose that the uncertain weights can be described by a fixed reference point and a perturbation region around the point; that is, the weight sets $W^{U}$ and $W^{L}$ can be given as follows: let $\bar{w}^{i} \in R^{m^{i}}$ be the reference point of $w^{i}$ and $C^{i} \in$ $R^{b^{i} \times m^{i}}$ be a coefficient matrix used to construct a perturbation region around $\bar{w}^{i}, i=U, L$, and then define the perturbation region around $\bar{w}^{i}$ as follows:

$$
\begin{array}{r}
W^{i}=\left\{w^{i} \in R^{m^{i}} \mid w^{i}=\bar{w}^{i}+\left(C^{i}\right)^{T} v^{i}, v^{i} \in V^{i} \subset R^{b^{i}}\right\}, \\
i=U, L,
\end{array}
$$

where $V^{i}, i=U, L$, are two uncertain sets which belong to

$$
\begin{aligned}
\widetilde{V}^{i} & =\left\{v^{i} \in R^{b^{i}} \mid \bar{w}^{i}+\left(C^{i}\right)^{T} v^{i}\right. \\
& \left.\geq 0,\left(e^{i}\right)^{T}\left(\bar{w}^{i}+\left(C^{i}\right)^{T} v^{i}\right)=1\right\}, \quad i=U, L,
\end{aligned}
$$

and here $e^{i}:=(1, \ldots, 1) \in R^{m^{i}}, i=U, L$. Note that the above method for defining uncertain parameters has been widely used in the robust optimization literature $[12,13]$.

Define

$$
V^{i}:=\left\{v^{i} \in \widetilde{V}^{i} \mid A^{i} v^{i}=\gamma^{i}, B^{i} v^{i} \geq c^{i}\right\}, \quad i=U, L,
$$

where $A^{i} \in R^{l^{i} \times b^{i}}, \gamma^{i} \in R^{l^{i}}, c^{i} \in R^{k^{i}}$, and $B^{i} \in R^{k^{i} \times b^{i}}$, $i=U, L$. We now present a reformulation for problem (5) with $W^{i}$ given as (7)-(9). Next, we show that the computation for a robust-weighted Nash equilibrium point of MBG can be casted as solving MPEC.

Lemma 4. Suppose the weight sets $W^{i}, i=U, L$, are given as (7)-(9), and then the robust-weighted bilevel optimization 
problem (5) is equivalent to the following bilevel optimization problem:

$$
\begin{array}{ll}
\min _{x^{i}, z_{j}^{i}, i=U, L ; j=1,2,3,4} & \left(\bar{w}^{U}\right)^{T} z_{3}^{U}+z_{4}^{U}-\left(\gamma^{U}\right)^{T} z_{1}^{U} \\
& -\left(c^{U}\right)^{T} z_{2}^{U} \\
\text { s.t. } \quad & C^{U} z_{3}^{U}+\left(A^{U}\right)^{T} z_{1}^{U}+\left(B^{U}\right)^{T} z_{2}^{U 2}=0 \\
& F^{U}\left(x^{U}, x^{L}\right)-z_{3}^{U}-z_{4}^{U} e \leq 0 \\
& z_{1}^{U} \in R^{l^{U}} \\
& z_{2}^{U} \in R_{+}^{k^{U}}, \\
& z_{3}^{U} \in R^{m^{U}}, \\
& z_{4}^{U} \in R \\
& G^{U}\left(x^{U}, x^{L}\right) \geq 0 \\
& H^{U}\left(x^{U}, x^{L}\right)=0 \\
& \left(x^{L}, z_{1}^{L}, z_{2}^{L}, z_{3}^{L}, z_{4}^{L}\right) \in S_{w}^{L}\left(x^{U}\right)
\end{array}
$$

where $S_{w}^{L}\left(x^{U}\right)$ signifies the set of solutions of the lower-level problem:

$$
\begin{aligned}
\min _{x^{L}, z_{j}^{L}, j=1,2,3,4} & \left(\bar{w}^{L}\right)^{T} z_{3}^{L}+z_{4}^{L}-\left(\gamma^{L}\right)^{T} z_{1}^{L}-\left(c^{L}\right)^{T} z_{2}^{L} \\
\text { s.t. } & C^{L} z_{3}^{L}+\left(A^{L}\right)^{T} z_{1}^{L}+\left(B^{L}\right)^{T} z_{2}^{L 2}=0 \\
& F^{L}\left(x^{L}, x^{U}\right)-z_{3}^{L}-z_{4}^{L} e \leq 0 \\
& G^{L}\left(x^{L}, x^{U}\right) \geq 0, \\
& H^{L}\left(x^{L}, x^{U}\right)=0 \\
& z_{1}^{L} \in R^{l^{L}}, \\
& z_{2}^{L} \in R_{+}^{k^{L}} \\
& z_{3}^{L} \in R^{m^{L}} \\
& z_{4}^{L} \in R .
\end{aligned}
$$

Proof. This lemma can be proven as follows. Given the leader's strategy $x^{U}$, it is easy to show that the follower's optimization problem (6) can be rewritten as

$$
\begin{array}{ll}
\min _{x^{L}, t} & t \\
\text { s.t. } & \max _{w^{L} \in W^{L}}\left(w^{L}\right)^{T} F^{L}\left(x^{L}, x^{U}\right) \leq t, \\
& G^{L}\left(x^{L}, x^{U}\right) \geq 0 \\
& H^{L}\left(x^{L}, x^{U}\right)=0 .
\end{array}
$$

For any given $\left(x^{U}, x^{L}\right)$, the left-hand side in the first inequality constraint in the above problem is equivalent to

$$
\begin{aligned}
\max _{v^{L}} & \left(C^{L} F^{L}\left(x^{L}, x^{U}\right)\right)^{T} v^{L}+\left(\bar{w}^{L}\right)^{T} F^{L}\left(x^{L}, x^{U}\right), \\
\text { s.t. } & \bar{w}^{L}+\left(C^{L}\right)^{T} v^{L} \geq 0, \\
& e^{T}\left(\bar{w}^{L}+\left(C^{L}\right)^{T} v^{L}\right)=1, \\
& A^{L} v^{L}=\gamma^{L}, \\
& B^{L} v^{L} \geq c^{L} .
\end{aligned}
$$

The corresponding dual problem is

$$
\begin{aligned}
\min _{z_{1}^{L}, z_{2}^{L}, z_{3}^{L}, z_{4}^{L}} & \left(\bar{w}^{L}\right)^{T} z_{3}^{L}+z_{4}^{L}-\left(\gamma^{L}\right)^{T} z_{1}^{L}-\left(c^{L}\right)^{T} z_{2}^{L}, \\
\text { s.t. } & C^{L} z_{3}^{L}+\left(A^{L}\right)^{T} z_{1}^{L}+\left(B^{L}\right)^{T} z_{2}^{L}=0 \\
& F^{L}\left(x^{L}, x^{U}\right)-z_{3}^{L}-z_{4}^{L} e \leq 0 \\
& z_{1}^{L} \in R^{l^{L}} \\
& z_{2}^{L} \in R_{+}^{k^{L}} \\
& z_{3}^{L} \in R^{m^{L}} \\
& z_{4}^{L} \in R,
\end{aligned}
$$

where " 0 " and " $e$ " are the zero and unit vectors with appropriate dimensions, respectively.

Therefore it follows from (12)-(14) and the strong duality theorem that the assertion of this theorem is true.

The above lemma shows the equivalence between the bilevel problems (5) and (10); that is, if $\left(x^{U}, x^{L}\right)$ is a robustweighted optimal solution to (5), then there is $\left(z_{j}^{i}\right)_{i=U, L ; j=1,2,3,4}$ such that $\left(x^{i}, z_{j}^{i}\right)_{i=U, L ; j=1,2,3,4}$ is an optimal solution to (10); otherwise if $\left(x^{i}, z_{j}^{i}\right)_{i=U, L ; j=1,2,3,4}$ is an optimal solution to (10), then $\left(x^{U}, x^{L}\right)$ is a robust-weighted optimal solution to (5). So, in what follows, we focus on the bilevel problem (10).

Given $x^{U}$, it is expected that a solution $\left(x^{L}, z_{j}^{L}\right)_{j=1,2,3,4}$ of the lower-level problem (11) is a stationary point of (11); that is, it solves the following Karush-Kuhn-Tucker (KKT) system:

$$
\begin{aligned}
& \nabla_{x^{L}} \Gamma \\
& =\nabla_{x^{L}} F^{L}\left(x^{L}, x^{U}\right) \lambda_{2}^{L}-\nabla_{x^{L}} G^{L}\left(x^{L}, x^{U}\right) \lambda_{4}^{L} \\
& +\nabla_{x^{L}} H^{L}\left(x^{L}, x^{U}\right) \lambda_{5}^{L}=0 ; \\
& \nabla_{z_{1}^{L}} \Gamma=-\gamma^{L}+A^{L} \lambda_{1}^{L}=0 ; \\
& \nabla_{z_{2}^{L}} \Gamma=-c^{L}+B^{L} \lambda_{1}^{L}-\lambda_{3}^{L}=0 ; \\
& \nabla_{z_{3}^{L}} \Gamma=\bar{w}^{L}+\left(C^{L}\right)^{T} \lambda_{1}^{L}-\lambda_{2}^{L}=0 ; \\
& \nabla_{z_{4}^{L}} \Gamma=1-e^{T} \lambda_{2}^{L}=0 ; \\
& H^{L}\left(x^{L}, x^{U}\right)=0
\end{aligned}
$$




$$
\begin{aligned}
& F^{L}\left(x^{L}, x^{U}\right)-z_{3}^{L}-z_{4}^{L} e \leq 0 \\
& \lambda_{2}^{L} \geq 0 \\
& \left(\lambda_{2}^{L}\right)^{T}\left(F^{L}\left(x^{L}, x^{U}\right)-z_{3}^{L}-z_{4}^{L} e\right)=0 \\
& z_{2}^{L} \geq 0 \\
& G^{L}\left(x^{L}, x^{U}\right) \geq 0 \\
& \lambda_{3}^{L} \geq 0 \\
& \lambda_{4}^{L} \geq 0 \\
& \left(\lambda_{3}^{L}\right)^{T} z_{2}^{L}=0 \\
& \left(\lambda_{4}^{L}\right)^{T} G^{L}\left(x^{L}, x^{U}\right)=0
\end{aligned}
$$

where $\Gamma$ is the Lagrangian of (11) defined as

$$
\begin{aligned}
\Gamma\left(\left(x^{L}, x^{U}\right),\left(z_{1}^{L}, z_{2}^{L}, z_{3}^{L}, z_{4}^{L}\right),\left(\lambda_{1}^{L}, \lambda_{2}^{L}, \lambda_{3}^{L}, \lambda_{4}^{L}, \lambda_{5}^{L}\right)\right) \\
:=\left(\bar{w}^{L}\right)^{T} z_{3}^{L}+z_{4}^{L}-\left(\gamma^{L}\right)^{T} z_{1}^{L}-\left(c^{L}\right)^{T} z_{2}^{L 2} \\
\quad+\left(\lambda_{1}^{L}\right)^{T}\left(C^{L} z_{3}^{L}+\left(A^{L}\right)^{T} z_{1}^{L}+\left(B^{L}\right)^{T} z_{2}^{L}\right) \\
\quad+\left(\lambda_{2}^{L}\right)^{T}\left(F^{L}\left(x^{L}, x^{U}\right)-z_{3}^{L}-z_{4}^{L} e\right)-\left(\lambda_{3}^{L}\right)^{T} z_{2}^{L} \\
\quad-\left(\lambda_{4}^{L}\right)^{T} G^{L}\left(x^{L}, x^{U}\right)+\lambda_{5}^{L} H^{L}\left(x^{L}, x^{U}\right) .
\end{aligned}
$$

Therefore replacing the lower level optimization problem (11) with (15) derives MPEC:

$$
\begin{aligned}
\min _{x^{i}, z_{j}^{i}, i=U, L ; j=1,2,3,4, \lambda_{k}^{L}, k=1, \ldots, 5} & \left(\bar{w}^{U}\right)^{T} z_{3}^{U}+z_{4}^{U}-\left(\gamma^{U}\right)^{T} z_{1}^{U}-\left(c^{U}\right)^{T} z_{2}^{U} \\
\text { s.t. } & C^{U} z_{3}^{U}+\left(A^{U}\right)^{T} z_{1}^{U}+\left(B^{U}\right)^{T} z_{2}^{U 2}=0 \\
& F^{U}\left(x^{U}, x^{L}\right)-z_{3}^{U}-z_{4}^{U} e \leq 0 \\
& z_{1}^{U} \in R^{l^{U}} \\
& z_{2}^{U} \in R_{+}^{k^{U}}, \\
& z_{3}^{U} \in R^{m^{U}}, \\
& z_{4}^{U} \in R \\
& G^{U}\left(x^{U}, x^{L}\right) \geq 0 \\
& H^{U}\left(x^{U}, x^{L}\right)=0 \\
& \left(x^{L}, z_{1}^{L}, \ldots, z_{4}^{L}, \lambda_{1}^{L}, \ldots, \lambda_{5}^{L}\right) \text { solves }\{(15)\} .
\end{aligned}
$$

This problem can be efficiently solved by the solver PATH [23]. We note that the PATH algorithm is originally described in [24]. This algorithm computes a stationary solution of MPEC (since it solves an equation related to the necessary optimality conditions). If for any given $x^{U}$ functions $F^{L}\left(\cdot, x^{U}\right)$ and $H^{L}\left(\cdot, x^{U}\right)$ are convex in $x^{L}$ and $G^{L}\left(\cdot, x^{U}\right)$ is concave in $x^{L}$, then under some constraint qualification an optimal solution of the bilevel optimization problem (10) is also a stationary solution of the MPEC (17) [25].

Theorem 5. Suppose the weight sets $W^{i}, i=U, L$, are given as (7)-(9). If for any given $x^{U}$ functions $F^{L}\left(\cdot, x^{U}\right)$ and $H^{L}\left(\cdot, x^{U}\right)$ are convex in $x^{L}$ and $G^{L}\left(\cdot, x^{U}\right)$ is concave in $x^{L}$, then under some constraint qualification an optimal solution of the bilevel optimization problem (5) is also a stationary solution of the MPEC (17).

Proof. The proof for this theorem follows directly from Lemma 4, the above discussions, and Proposition 5 [25].

\section{An Application}

4.1. Model Description. In this section, we model the multicriteria competition in a supply chain of $M$ manufacturers who cooperatively decide on volume to supply $P$ products to $N$ risk-averse retailers who also cooperatively decide on 
volume to satisfy uncertain consumer demand. Manufacturers have two objectives (i.e., to maximize their profit and to minimize their cost of products supplied simultaneously) by choosing the supply quantity for each of $P$ products in the anticipation of the order quantities of the retailers and also anticipating of the wholesale prices resulting from the market clearing conditions. Retailers have also two objectives (i.e., to maximize the expected utility from retail sales and to minimize the risk expressed as the standard deviation of the profit) by deciding the wholesale market order quantity for each of $P$ products.

The retail demand satisfies the following stochastic linear inverse demand function:

$$
\widehat{p}=(\widehat{a}-\widehat{B} \widehat{x}) \xi
$$

where $\widehat{p}=\left(p_{1}, \ldots, p_{N}\right)^{T} \in R^{N P}$ is the aggregate price vector, $p_{j}$ is the vector of the $j$ th retailer prices with $p_{j}=\left(p_{j 1}\right.$, $\left.\ldots, p_{j P}\right), p_{j k}$ is the price of the $j$ th retailer for the $k$ th product, and $\xi=\sum_{l=1}^{r} \lambda_{l} \xi_{l}$ with $\xi_{l}$ as a positive scalar random variable with mean $\mu_{l}$ and finite standard deviation $\sigma_{l}, \lambda_{l} \geq 0$, and $\sum_{l=1}^{r} \lambda_{l}=1$. The vector $\hat{a}=\left(a_{1}, \ldots, a_{N}\right)^{T} \in R^{N P}$ with $a_{j}=$ $\left(a_{j 1}, \ldots, a_{j P}\right)$ gives the prices that the consumers would be willing to pay if the retail market supply was 0 and $\xi$ is a finite number. The matrix $\widehat{B} \in R^{N P \times N P}$ is the positive definite matrix of inverse demand sensitivities which can be expressed as

$$
\left(\begin{array}{cccc}
H_{1} & G_{12} & \cdots & G_{1 N} \\
G_{21} & H_{2} & \cdots & G_{2 N} \\
\vdots & \vdots & \ddots & \vdots \\
G_{N 1} & G_{N 2} & \cdots & H_{N}
\end{array}\right)
$$

with $H_{j} \in R^{P \times P}, j=1, \ldots, N, G_{j \kappa} \in R^{P \times P}$, and $j, \kappa=$ $1, \ldots, N, j \neq \kappa$. The vector $\widehat{x}=\left(x_{1}, \ldots, x_{N}\right)^{T} \in R^{N P}$ is the aggregate retailer order vector, where $x_{j}$ is the vector of the $j$ th retailer order vector $x_{j}=\left(x_{j 1}, \ldots, x_{j P}\right)$.

Retailers make their order quantity decision as follows:

$$
\begin{aligned}
\max & -\sum_{j=1}^{N}\left(x_{j}^{T} \pi+x_{j}^{T}\left(a_{j}-\widehat{B}_{j} \cdot \widehat{x}\right) \mathbb{E}[\xi]\right), \\
\min & \sum_{j=1}^{N} x_{j}^{T}\left(a_{j}-\widehat{B}_{j} \cdot \widehat{x}\right) \sigma(\xi), \\
\text { s.t. } & x_{j} \geq 0, \\
& j=1, \ldots, N,
\end{aligned}
$$

where $\pi=\left(\pi_{1}, \ldots, \pi_{P}\right)$ is the vector of wholesale prices and $\widehat{B}_{j} .=\left(G_{j 1}, \ldots, H_{j}, \ldots, G_{j N}\right)$, for $j$ th retailer whose first objective is to maximize their mean profit and second objective is to minimize their standard deviation for the stochastic demand. We assume that the weight vector to the two objectives $x_{j}^{T} \pi+x_{j}^{T}\left(a_{j}-\widehat{B}_{j} \widehat{x}\right) \mathbb{E}[\xi]$ and $x_{j}^{T}\left(a_{j}-\widehat{B}_{j} . \widehat{x}\right) \sigma(\xi)$ in $(20)$ is $w_{j}^{L}:=\left(w_{j 1}^{L}, w_{j 2}^{L}\right) \in R_{++}^{2}$.
Manufacturers choose their supply quantity to maximize their profit in the wholesale market, anticipating the market clearing wholesale market price as well as the retailer order quantities. Mathematically, manufacturers face the following decision problem:

$$
\begin{array}{ll}
\min & \sum_{i=1}^{M}\left(w_{i 1}^{U} c_{i}^{T} y_{i}-w_{i 2}^{U} \pi^{T} y_{i}\right), \\
\text { s.t. } & \sum_{k=1}^{M} y_{k}=\sum_{j=1}^{N} x_{j}(v), \\
& y_{i} \geq 0, \\
& i=1, \ldots, M, \\
& v \in R^{P},
\end{array}
$$

where $c_{i} \in R^{P}$ is the $i$ th manufacturer unit cost, $y_{i} \in R^{P}$ is the supply quantity of the $i$ th manufacturer and $x_{j}(\pi): R^{P} \rightarrow$ $R^{P}$ is the $j$ th retailer order quantity for a given wholesale price $\pi, w_{i}^{U}:=\left(w_{i 1}^{U}, w_{i 2}^{U}\right) \in R_{++}^{2}$ is the weight vector to manufacturer $i$ 's profit and cost, and the first constraint is the wholesale market clearing conditions. We assume that all the uncertainty exists in the retail market and that the wholesale market is deterministic except for the weight choice. The reason for this is that the demand uncertainty in the retail demand function is realized when all wholesale market related decisions are made in our model. We also assume that the weight vector $w_{i}^{U}$ is uncertain and belongs to a known convex and compact set. By this assumption, we can utilize the proposed robust-weighted approach.

4.2. Numerical Results. We now detail the numerical tests for finding a robust-weighted manufacturer-retailer equilibrium by using the numerical solver PATH [23]. We present numerical results for the robust-weighted manufacturer-retailer equilibrium of models in the decentralized supply chain with finite weights and general polyhedral weight regions, respectively. Suppose $M=2, N=2$, and $P=2$, that is, two manufacturers, two retailers, and two products. We assume that $\mathbb{E}[\xi]=1$ and $\sigma(\xi)=0.5$; that is, the mean and the standard deviation of the stochastic variable $\xi$ are 1 and 0.5 , respectively. We give the following assumption for the parameters in supply chain:

$$
\begin{aligned}
a_{1} & :=\left[\begin{array}{l}
9 \\
11
\end{array}\right] ; \\
a_{2} & :=\left[\begin{array}{l}
7 \\
10
\end{array}\right] ; \\
c_{1} & :=\left[\begin{array}{l}
0.6 \\
0.6
\end{array}\right] ; \\
c_{2} & :=\left[\begin{array}{l}
0.5 \\
0.5
\end{array}\right] \\
H_{j} & =G_{j \kappa}:=\left[\begin{array}{cc}
1 & 0.5 \\
0.5 & 1
\end{array}\right], \quad j, \kappa=1,2, j \neq \kappa .
\end{aligned}
$$


TABLE 1: Numerical test results with finite weights for $g_{1}^{1} / g_{2}^{1}=0.3$ and $g_{1}^{2} / g_{2}^{2}=0.5$.

\begin{tabular}{lcccc}
\hline$f_{1}^{1} / f_{2}^{1}$ & $f_{1}^{2} / f_{2}^{2}$ & $\hat{x}$ & $\hat{y}$ & $\pi$ \\
\hline 0.7 & 0.8 & $(0.583,0.527,0,1.654)$ & $(0,0,0.583,2.181)$ & $(1.851,2.203)$ \\
0.7 & 1.28 & $(0,0,0.393,1.893)$ & $(0,0,0.393,1.893)$ & $(2.633,3.547)$ \\
0.7 & 1.69 & $(0,0,0.430,1.930)$ & $(0,0,0.430,1.930)$ & $(2.964,4.021)$ \\
1 & 1.28 & $(0.833,0.923,0,1.554)$ & $(0,0,0.833,2.476)$ & $(2.817,3.384)$ \\
1 & 0.8 & $(0.833,1.833,0,0)$ & $(0,0,0.833,1.833)$ & $(2.750,3.250)$ \\
1 & 1.69 & $(0.833,0.513,0,2.000)$ & $(0,0,0.833,2.513)$ & $(2.910,3.570)$ \\
1.5 & 1.69 & $(0.917,1.385,0,1.170)$ & $(0,0,0.917,2.555)$ & $(3.465,4.096)$ \\
1.5 & 1.28 & $(0.917,1.801,0,0.717)$ & $(0,0,0.917,2.518)$ & $(3.338,3.843)$ \\
1.5 & 0.8 & $(0.917,1.917,0,0)$ & $(0,0,0.917,1.917)$ & \\
\hline
\end{tabular}

TABLE 2: Numerical test results with finite weights for $g_{1}^{1} / g_{2}^{1}=0.3$ and $g_{1}^{2} / g_{2}^{2}=1.2$.

\begin{tabular}{lcccc}
\hline$f_{1}^{1} / f_{2}^{1}$ & $f_{1}^{2} / f_{2}^{2}$ & $\widehat{x}$ & $\widehat{y}$ & $\pi$ \\
\hline 0.7 & 0.8 & $(0.769,0.664,0,1.751)$ & $(0,0,0.769,2.415)$ & $(1.692,2.044)$ \\
0.7 & 1.28 & $(0.278,0,0.814,1.980)$ & $(0,0,1.092,1.980)$ & $(1.897,3.099)$ \\
0.7 & 1.69 & $(0,0,0.505,2.005)$ & $(0,0,0.505,2.005)$ & $(2.805,3.862)$ \\
1 & 1.28 & $(0.939,0.995,0,1.622)$ & $(0,0,0.939,2.617)$ & $(2.658,3.625)$ \\
1 & 0.8 & $(0.939,1.939,0,0)$ & $(0,0,0.939,1.939)$ & $(2.591,3.091)$ \\
1 & 1.69 & $(0.939,0.597,0,2.045)$ & $(0,0,0.939,2.642)$ & $(2.751,3.411)$ \\
1.5 & 1.69 & $(0.996,1.429,0,1.241)$ & $(0,0,0.996,2.670)$ & $(3.305,3.937)$ \\
1.5 & 1.28 & $(0.996,1.833,0,0.812)$ & $(0,0,0.996,2.654)$ & $(3.179,3.684)$ \\
1.5 & 0.8 & $(0.966,1.996,0,0)$ & $(0,0,0.996,1.996)$ & $(3.341,4.008)$ \\
\hline
\end{tabular}

Analytical Hierarchy Process (for brief AHP) [26] is used to assign weights for the two objectives in models (20) and (21). We assume that retailer $j$ is unsure about the relative importance of $f_{1}^{j}(\widehat{x}):=x_{j}^{T} \pi-x_{j}^{T}\left(a_{j}-\widehat{B}_{j} . \widehat{x}\right) \mathbb{E}[\xi]$ and $f_{2}^{j}(\widehat{x}):=x_{j}^{T}\left(a_{j}-\widehat{B}_{j} \cdot \widehat{x}\right) \sigma(\xi)$ for any $j=1,2$. Consequently, we first consider that there are finite weights to quantify the relative importance for the two objective functions. Then we generalize the finite weights to the weight set given by a polytope. We first suppose that retailers allow three possible scale indices for the two objective functions as $f_{1}^{1} / f_{2}^{1}=0.7,1$, or 1.5 and $f_{1}^{2} / f_{2}^{2}=0.8,1.28$, or 1.69. Similar to the assumption about the retailers' weights, we also assume that manufacturer $i$ is also unsure about the relative importance of $g_{1}^{i}:=-\pi^{T} y_{i}$ and $g_{2}^{i}:=c_{i}^{T} y_{i}, i=1,2$. As such, manufacturers allow for two possible scale indices to the two objective functions as $g_{1}^{1} / g_{2}^{1}=0.3$ or 1 and $g_{2}^{2} / g_{2}^{2}=0.5$ or 1.2 .

The numerical results are reported in Tables 1-4, where $(\hat{x}, \hat{y}, \pi)$ (manufacturer-retailer equilibriums) are the weighted Nash equilibrium by using the weighted approach [9], and here the vector $\hat{y}=\left(y_{1}, \ldots, y_{M}\right)^{T} \in R^{M P}$ is the aggregate manufacturer supply vector. The robust-weighted Nash equilibrium (RWNE) with finite weights is reported in the last row of Table 4 . There are different weighted manufacturerretailer equilibriums for a different choice of the weights. The robust-weighted retailer equilibrium is computed by using the weight region generated as the convex hull of the nine weight vectors. It is easy to see that the robust-weighted approach presents a unique equilibrium which mitigates the conflict with different weighted manufacturer-retailer equilibria for a different choice of the weights in Tables 1-4. We note that, compared with the existing weighted approach [9], our method is more "robust" in that there are different solutions for choosing different weights by using weighted approach [9], but there is a unique solution by using our approach.

The managerial impact of the proposed method is as follows. The proposed approach overcomes several shortcomings for the current approaches to the multicriteria competition in a supply chain. There is no clear guidance to a decision-maker (manufacturers or retailers) to choose a solution among (possibly a very large number of) generated Pareto optimal solutions. However, there is a unique solution by using our approach. In the multicriteria competition in a supply chain, since different decision-maker (manufacturers or retailers) may have different opinions, the relative weights given by different decision-maker can differ significantly. Furthermore, there may be ambiguity in the weights given by a decision-maker (manufacturer or retailer), because it is often difficult to give relative weights for each objective. 
TABLE 3: Numerical test results with finite weights for $g_{1}^{1} / g_{2}^{1}=1$ and $g_{1}^{2} / g_{2}^{2}=1.2$.

\begin{tabular}{lcccr}
\hline$f_{1}^{1} / f_{2}^{1}$ & $f_{1}^{2} / f_{2}^{2}$ & $\hat{x}$ & $\hat{y}$ & $\pi$ \\
\hline 0.7 & 0.8 & $(0.924,0.778,0,1.832)$ & $(0,0,0.924,2.610)$ & $(1.560,1.911)$ \\
0.7 & 1.28 & $(0.515,0,0.768,2.053)$ & $(0,0,1.283,2.053)$ & $(1.764,2.967)$ \\
0.7 & 1.69 & $(0.255,0,1.036,2.068)$ & $(0,0,1.291,2.068)$ & $(1.834,3.310)$ \\
1 & 1.28 & $(1.028,1.055,0,1.697)$ & $(0,0,1.028,2.733)$ & $(2.525,3.092)$ \\
1 & 0.8 & $(1.028,2.028,0,0)$ & $(0,0,1.028,2.028)$ & $(2.458,2.958)$ \\
1 & 1.69 & $(1.028,0.667,0,2.082)$ & $(0,0,1.028,2.749)$ & $(3.173,3.804)$ \\
1.5 & 1.69 & $(1.062,1.466,0,1.300)$ & $(0,0,1.062,2.766)$ & $(3.046,3.551)$ \\
1.5 & 1.28 & $(1.062,1.860,0,0.891)$ & $(0,0,1.062,2.751)$ & $(3.208,3.875)$ \\
1.5 & 0.8 & $(1.063,2.062,0,0)$ & $(0,0,1.063,2.062)$ & \\
\hline
\end{tabular}

TABLE 4: Numerical test results with finite weights for $g_{1}^{1} / g_{2}^{1}=1$ and $g_{1}^{2} / g_{2}^{2}=0.5$.

\begin{tabular}{lcccc}
\hline$f_{1}^{1} / f_{2}^{1}$ & $f_{1}^{2} / f_{2}^{2}$ & $\hat{x}$ & $\hat{y}$ & $\pi$ \\
\hline 0.7 & 0.8 & $(0.817,0.699,0,1.776)$ & $(0.817,2.475,0,0)$ & $(1.651,2.003)$ \\
0.7 & 1.28 & $(0.351,0,0.800,2.003)$ & $(1.151,2.003,0,0)$ & $(1.856,3.059)$ \\
0.7 & 1.69 & $(0,0,0.525,2.025)$ & $(0.525,2.025,0,0)$ & $(2.764,3.821)$ \\
1 & 1.28 & $(0.967,1.013,0,1.639)$ & $(0.967,2.653,0,0)$ & $(2.617,3.184)$ \\
1 & 0.8 & $(0,2.867,0,1.167)$ & $(0,3.033,0,0)$ & $(2.710,3.370)$ \\
1 & 1.69 & $(0.967,0.619,0,2.056)$ & $(0.967,2.675,0,0)$ & $(3.265,3.896)$ \\
1.5 & 1.69 & $(1.017,1.440,0,1.259)$ & $(1.017,2.700,0,0)$ & $(4.859,3.643)$ \\
1.5 & 1.28 & $(0,2.650,0,0.836)$ & $(0,3.186,0,0)$ & $(3.300,3.967)$ \\
1.5 & 0.8 & $(1.017,2.017,0,0)$ & $(1.017,2.017,0,0)$ & $(1.851,2.203)$ \\
& $($ RWNE) & $(0.583,0.527,0,1.654)$ & $(0,0,0.583,2.181)$ & \\
\hline
\end{tabular}

TABLE 5: Numerical test results with general polytope weight regions.

\begin{tabular}{lccr}
\hline$\delta$ & $\hat{x}$ & $\hat{y}$ & $\pi$ \\
\hline 0 & $(0.778,1.444,0.778,1.444)$ & $(0,0,1.556,2.889)$ & $(2.750,3.250)$ \\
0.007 & $(0.777,1.444,0.777,1.444)$ & $(0,0,1.544,2.887)$ & $(2.748,3.248)$ \\
0.02 & $(0.776,1.442,0.776,1.442)$ & $(0,0,1.541,2.884)$ & $(2.745,3.245)$ \\
0.05 & $(0.772,1.439,0.772,1.439)$ & $(0,0,1.544,2.878)$ & $(2.737,3.237)$ \\
0.1 & $(0.767,1.433,0.767,1.433)$ & $(0,0,1.533,2.867)$ & $(2.725,3.225)$ \\
0.5 & $(0.722,1.389,0.722,1.389)$ & $(0,0,1.444,2.778)$ & $(2.625,3.125)$ \\
\hline
\end{tabular}

For the general polytope weight region, we suppose the reference point $\bar{w}^{j}$ and matrices $C_{i}^{U}$ and $C_{j}^{L}$ are defined as follows: $\bar{w}_{i}^{U}:=(1 / 2,1 / 2), i=1,2, \bar{w}_{j}^{L}:=(1 / 3,2 / 3), j=1,2$, and

$$
\begin{array}{ll}
C_{i}^{U}:=\left[\begin{array}{ll}
1 & 2 \\
1 & 2
\end{array}\right], \quad i=1,2 \\
C_{j}^{L}:=\left[\begin{array}{ll}
1 & 0 \\
1 & 1
\end{array}\right], \quad j=1,2 .
\end{array}
$$

The perturbation subsets are defined as

$$
\begin{array}{ll}
V_{i}^{U}:=\left\{v_{i}^{U} \in R^{2} \mid e^{T} v_{i}^{U} \leq 1-\delta\right\}, & i=1 ; \\
V_{j}^{L}:=\left\{v_{j}^{L} \in R^{2} \mid e^{T} v_{j}^{L} \leq 1-\delta\right\}, \quad j=1,2,
\end{array}
$$

where $\delta$ is a parameter defined in $[0,1)$. With the above data the robust-weighted manufacturer-retailer equilibrium with different $\delta$ can be calculated by utilizing the method developed in Section 5. The stability results are shown in Table 5 which contains small data errors due to the small changes in $\delta$.

\section{Conclusion}

This paper introduces a robust-weighted approach to multicriteria bilevel problems. The computation of the robustweighted Pareto optimal point, with the polyhedral weight sets, can be equivalently transformed into solving MPEC. As an application, the new approach is used to analyze supply chain multicriteria bilevel competition. The numerical 
tests show that our method is more robust than existing approaches and a unique robust-weighted Pareto optimum can always be obtained for general polyhedral weight sets.

\section{Conflicts of Interest}

The authors declare that they have no conflicts of interest.

\section{Acknowledgments}

The work is supported by The Program for Professor of Special Appointment (Eastern Scholar) at Shanghai Institutions of Higher Learning (no. TP2014043). The work is also supported by Natural Scientific Foundation of China (no. 71401107; no. 71571055).

\section{References}

[1] S. Dempe, "Annotated bibliography on bilevel programming and mathematical programs with equilibrium constraints," Optimization, vol. 52, no. 3, pp. 333-359, 2003.

[2] H. Bonnel, "Optimality conditions for the semivectorial bilevel optimization problem," Pacific Journal of Optimization, vol. 2, no. 3, pp. 447-467, 2006.

[3] H. Bonnel and J. Morgan, "Semivectorial bilevel optimization problem: penalty approach," Journal of Optimization Theory and Applications, vol. 131, no. 3, pp. 365-382, 2006.

[4] B. Liu, Z. Wan, J. Chen, and G. Wang, "Optimality conditions for pessimistic semivectorial bilevel programming problems," Journal of Inequalities and Applications, vol. 2014, article no. 41, 2014.

[5] Y. Zheng and Z. P. Wan, "A solution method for semivectorial bilevel programming problem via penalty method," Journal of Applied Mathematics and Computing, vol. 37, no. 1-2, pp. 207219, 2011.

[6] A. Ben-Tal, A. Goryashko, E. Guslitzer, and A. Nemirovski, "Adjustable robust solutions of uncertain linear programs," Mathematical Programming, vol. 99, no. 2, pp. 351-376, 2004.

[7] V. Jeyakumar, G. M. Lee, and G. Li, "Characterizing robust solution sets of convex programs under data uncertainty," Journal of Optimization Theory and Applications, vol. 164, no. 2, pp. 407-435, 2015.

[8] M. Ehrgott, Multicriteria Optimization, Springer, Berlin, Germany, 2nd edition, 2005.

[9] S. Y. Wang, "Existence of a Pareto equilibrium," Journal of Optimization Theory and Applications, vol. 79, no. 2, pp. 373384, 1993.

[10] J. Yu and G. X. Yuan, "The study of Pareto equilibria for multiobjective games by fixed point and Ky Fan minimax inequality methods," Computers \& Mathematics with Applications, vol. 35, no. 9, pp. 17-24, 1998.

[11] E. Tsiporkova and V. Boeva, "Multi-step ranking of alternatives in a multi-criteria and multi-expert decision making environment," Information Sciences, vol. 176, no. 18, pp. 2673-2697, 2006.

[12] P. J. H. Schoemaker and C. C. Waid, "An experimental comparison of different approaches to determining weights in additive utility models," Management Science, vol. 28, no. 2, pp. 182-196, 1982.
[13] M. Weber and K. Borcherding, "Behavioral influences on weight judgments in multiattribute decision making," European Journal of Operational Research, vol. 67, no. 1, pp. 1-12, 1993.

[14] Y. Yin, "Multi-objective bilevel optimization for transportation planning and management problems," Journal of Advanced Transportation, vol. 36, no. 1, pp. 93-105, 2002.

[15] K. Deb and A. Sinha, Solving Bilevel Multi-Objective Optimization Problems Using Evolutionary Algorithms, Evolutionary Multi-Criterion Optimization, Springer, 2009.

[16] C. O. Pieume, P. Marcotte, L. P. Fotso, and P. Siarry, "Solving bilevel linear multiobjective programming problems," American Journal of Operations Research, vol. 1, no. 4, pp. 214-219, 2011.

[17] G. Eichfelder, "Multiobjective bilevel optimization," Mathematical Programming. A Publication of the Mathematical Programming Society, vol. 123, no. 2, pp. 419-449, 2010.

[18] Y. Ji, S. J. Qu, and Z. S. Yu, "Bi-level multi-objective optimization model for last mile delivery using a discrete approach," Journal of Difference Equations and Applications, 2016.

[19] H. I. Calvete and C. Galé, "Linear bilevel programs with multiple objectives at the upper level," Journal of Computational and Applied Mathematics, vol. 234, no. 4, pp. 950-959, 2010.

[20] H. I. Calvete and C. Galé, "On linear bilevel problems with multiple objectives at the lower level," Omega, vol. 39, no. 1, pp. 33-40, 2011.

[21] J. Hu and S. Mehrotra, "Robust and stochastically weighted multiobjective optimization models and reformulations," Operations Research, vol. 60, no. 4, pp. 936-953, 2012.

[22] J.-B. Jian, J.-L. Li, and X.-D. Mo, "A strongly and superlinearly convergent SQP algorithm for optimization problems with linear complementarity constraints," Applied Mathematics and Optimization, vol. 54, no. 1, pp. 17-46, 2006.

[23] M. C. Ferris and T. S. Munson, "Complementarity problems in GAMS and the PATH solver," Journal of Economic Dynamics \& Control, vol. 24, no. 2, pp. 165-188, 2000.

[24] S. P. Dierkse, Robust solution of mixed complementarity problems [Ph.D. thesis], University of Wisconsin-Madison, Madison, Wis, USA, 1994.

[25] X. Hu and D. Ralph, "Using EPECs to model bilevel games in restructured electricity markets with locational prices," Operations Research, vol. 55, no. 5, pp. 809-827, 2007.

[26] T. L. Saaty, "The modern science of multicriteria decision making and its practical applications: the AHP/ANP approach," Operations Research, vol. 61, no. 5, pp. 1101-1118, 2013. 


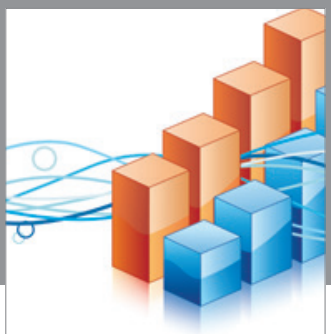

Advances in

Operations Research

vatem alat4

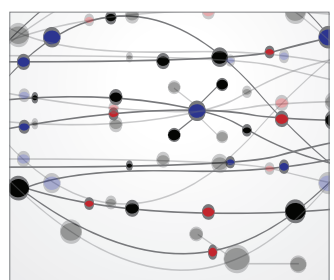

\section{The Scientific} World Journal
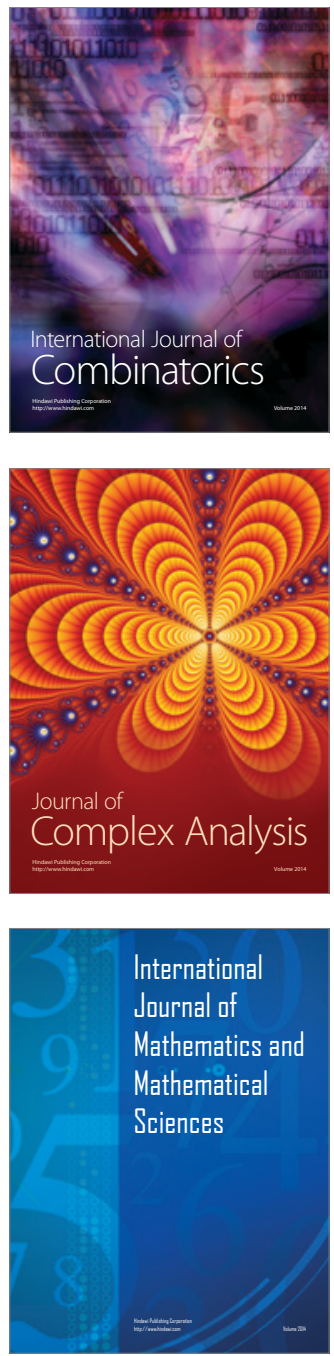
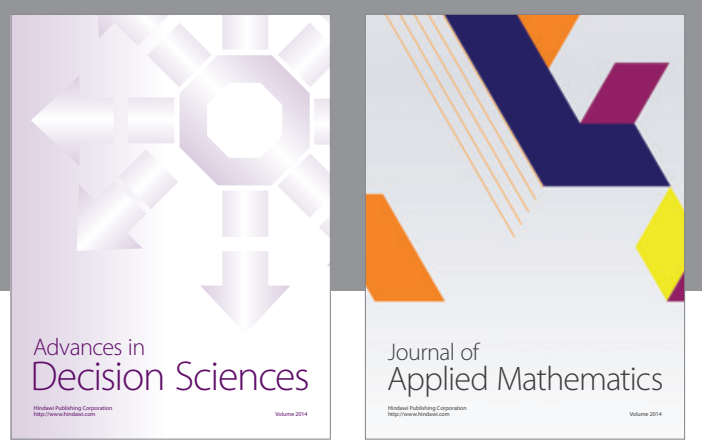

Algebra

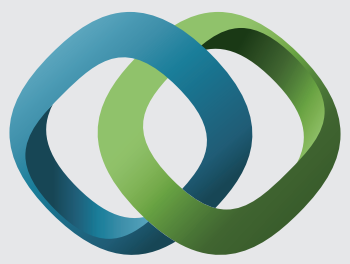

\section{Hindawi}

Submit your manuscripts at

https://www.hindawi.com
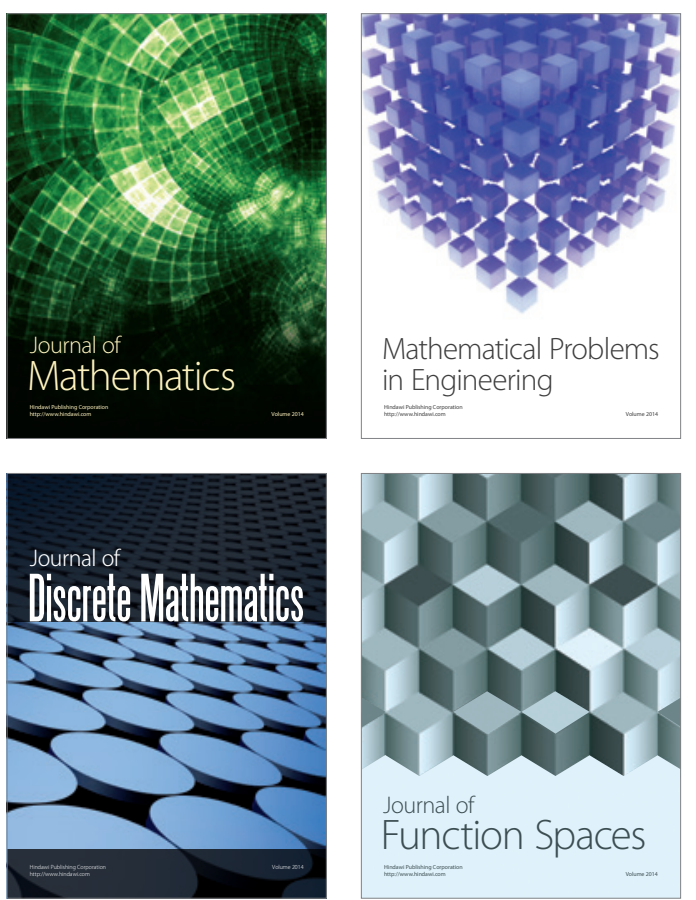

Mathematical Problems in Engineering
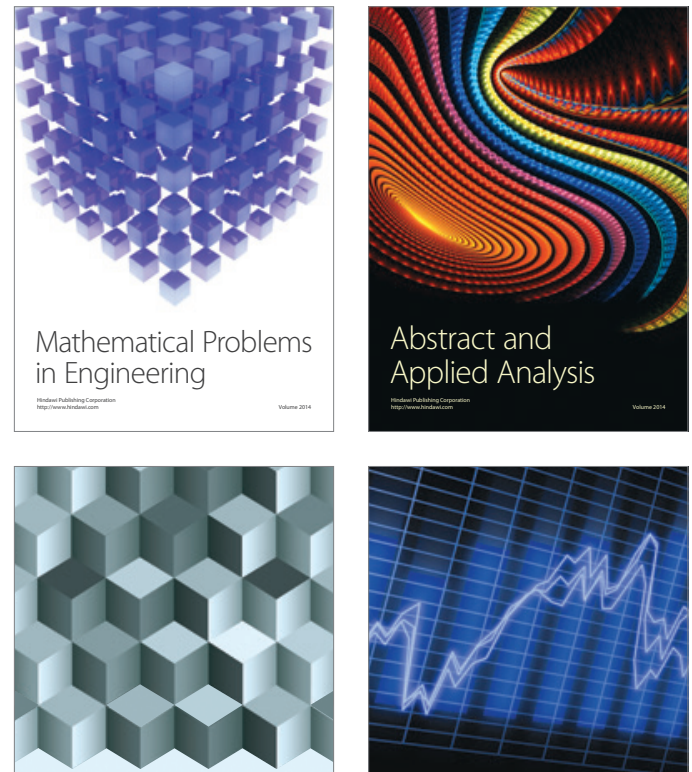

Journal of

Function Spaces

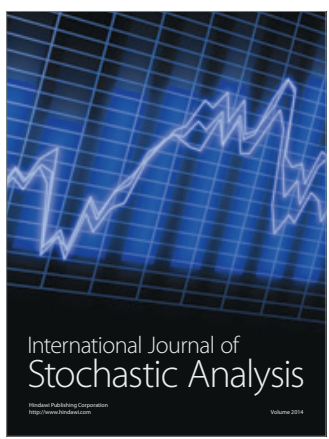

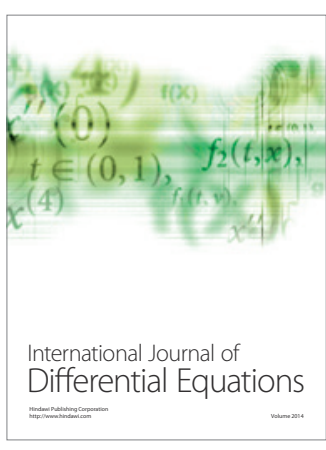
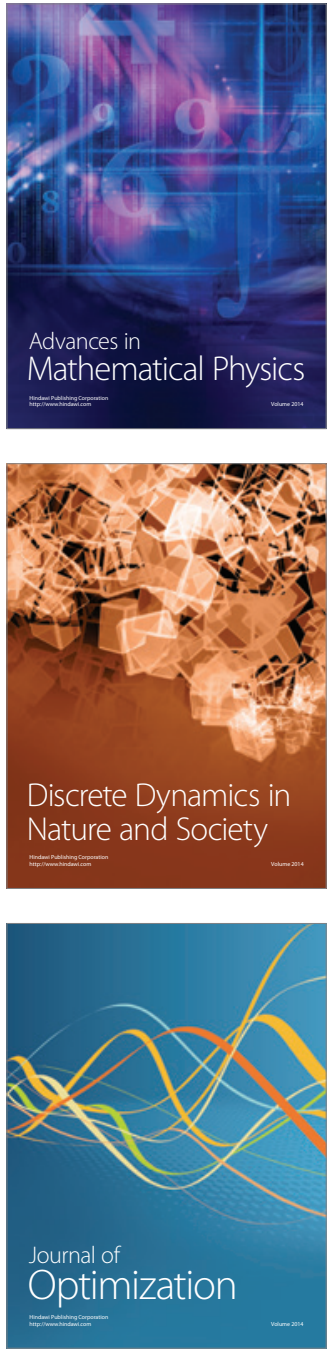